\title{
Origem e distribuição do plexo braquial de Saimiri sciureus ${ }^{1}$
}

\author{
Elenara B. Araújo², Ana R. Lima², Luane L. Pinheiro², José A.P.C. Muniz³ \\ Aline Imbeloni ${ }^{3}$ e Érika Branco ${ }^{2 *}$
}

\begin{abstract}
Araújo E.B., Lima A.R., Pinheiro L.L., Muniz J.A.P.C., Imbeloni A. \& Branco E. 2012. [Origin and distribution of the brachial plexus of Saimiri sciureus.] Origem e distribuição do plexo braquial de Saimiri sciureus. Pesquisa Veterinária Brasileira 32(12):13511354. Setor de Anatomia Descritiva dos Animais Domésticos e Selvagens do Instituto de Saúde e Produção Animal, Faculdade de Medicina Veterinária da Universidade Federal Rural da Amazônia, Campus de Belém, PA 66077-530, Brazil. E-mail: ebranco.ufra@gmail.com

The authors described the origin and composition of the brachial plexus of four Saimiri sciureus, from the National Primate Center (Cenp), Ananindeua/PA, which were fixed with formaldehyde and dissected. Findings revealed that the brachial plexus of this species is composed by nervous fibers from the roots of cervical vertebrae C4 to C8 and thoracic vertebrae T1, and organized into four branchs. Each branch has formed a nerve or a group of nerves, the origin was varied between animals, mostly were found the cranial trunk originate the subclavian nerve; the medium-cranial originate the suprascapular, subscapular, part of radial and in some cases the axillary, musculocutaneous and median nerves; the medium-caudal trunk originate part of radial nerve and in some cases the axillary, musculocutaneous, median, thoracodorsal, ulnar and medial cutaneous of forearm nerves, the last two nerves also originate from the caudal trunk.
\end{abstract}

INDEX TERMS: Nerves, brachial plexus, nonhuman primate, Saimiri sciureus.

RESUMO.- Os autores descreveram a origem e composição do plexo braquial de quatro Saimiri sciureus, pertencentes ao Centro Nacional de Primatas (Cenp), Ananindeua/PA, os quais foram fixados com formaldeído e dissecados. Os achados revelaram que o plexo braquial desta espécie é constituído por fibras neurais provenientes da união das raízes dorsais e ventrais das vértebras cervicais $\mathrm{C} 4$ a $\mathrm{C} 8$ e torácica $\mathrm{T} 1$, e organizado em quatro troncos. Cada tronco formou um nervo ou um grupo de nervos, cuja origem variou entre os animais; na maioria, foi encontrado o tronco cranial originando o nervo subclávio, o tronco médio-cranial dando origem aos nervos supraescapular, subescapular, parte do radial, e em alguns casos ao nervo axilar, nervo musculocu-

\footnotetext{
${ }^{1}$ Recebido em 15 de março de 2012.

Aceito para publicação em 6 de setembro de 2012.

${ }^{2}$ Instituto de Saúde e Produção Animal (ISPA), Faculdade de Medicina Veterinária, Universidade Federal Rural da Amazônia (UFRA), Av. Presidente Tancredo Neves 2501, Bairro Montese, Belém, PA 66077-530, Brasil. *Autor para correspondência: ebranco.ufra@gmail.com

${ }^{3}$ Instituto de Saúde e Produção Animal (ISPA), Faculdade de Medicina Veterinária, UFRA, Campus de Belém, PA.

${ }^{4}$ Centro Nacional de Primatas (Cenp), Instituto Evandro Chagas (IEC), Secretaria de Vigilância em Saúde (SVS), Ministério a Saúde (MS), BR 316 Km 7, Cx. Postal 44, Ananindeua, PA 67030-000, Brasil.
}

tâneo e ao nervo mediano; o tronco médio-caudal formou parte do nervo radial, e em alguns casos os nervos axilar, nervo musculocutâneo, nervo mediano, nervo toracodorsal, nervo ulnar e nervo cutâneo medial do antebraço, sendo os dois últimos também originados no tronco caudal.

TERMOS DE INDEXAÇÃO: Nervos, plexo braquial, primata não humano, macaco-de-cheiro, Saimiri sciureus.

\section{INTRODUÇÃO}

Os cebídeos constituem a maioria dos primatas neotropicais e dentre estes se destaca a subfamília Saimiriinae, a que pertence Saimiri sciureus Linnaeus, 1758, uma das espécies brasileiras mais utilizadas como modelo biológico (Vaughan 1985, Aurichio 1995), sendo considerado um pequeno primata do Novo Mundo que habita as florestas tropicais da Bacia Amazônica (Barcellos et al. 2011). Conhecidos como macacos-de-cheiro, vivem no topo das árvores altas, a 30 ou $40 \mathrm{~m}$ do chão, frequentemente em bandos de centenas de indivíduos. Considerados frutívoros e insetívoros, são esbeltos, ágeis e uma de suas principais características é cauda muito espessa. A espécie $S$. sciureus, diferente de outros primatas neotropicais, apresenta uma sazonalidade bem definida (Dukelow 1978). 
S. sciureus é o menor primata da família Cebidae, no qual os machos são pouco menores que as fêmeas. A pelagem é curta, espessa, macia e colorida brilhante. A pele dos lábios, incluindo a área em volta das narinas, é negra e quase desprovida de pêlos. A coloração mais comum é: branco ao redor dos olhos e das orelhas (com tufos brancos) e o pescoço bem como a parte dorsal da cabeça variam de negro a cinzento; mãos, pés e antebraços são amarelo-laranja; os ombros e os membros pélvicos, cobertos de cinza. A cauda é bicolor como o corpo, adornada ligeiramente na ponta, e não preênsil. Possuem unhas em vez de garras, os dentes são sexualmente dimórficos, na medida em que os machos possuem grandes caninos superiores. As marcações e coloração dos sexos e de animais de diferentes idades são semelhantes (Costello et al. 1997).

Geograficamente estão distribuídos também em vários países: Brasil, Colômbia, Guiana Francesa, Guiana, Suriname e Venezuela, Paraguai, Costa Rica e Panamá (Emmons \& Feer 1997).

O plexo braquial, objeto de nosso estudo, de forma geral supre quase todas as estruturas do membro torácico, sendo formado pelos últimos três nervos cervicais e os dois primeiros torácicos; o segundo e quinto nervos cervicais às vezes participa. Ao chegar na região axilar, o plexo braquial rapidamente se divide em ramos periféricos, que divergem na direção de seus destinos isolados (Getty 1986, Evans \& La Hunta 1994, Dyce 2004, Frandson et al. 2005), porém, não há registros se a distribuição do plexo braquial no $S$. sciureus se dá da mesma forma. Desta forma este trabalho tem como objetivo descrever a origem e distribuição do plexo braquial nesta espécie, a fim de contribuir com a morfologia comparativa.

Toda nomenclatura adotada foi baseada na Nomenclatura Atômica Veterinária (International Committee on Veterinary Gross Anatomical Nomenclature, 2005).

\section{MATERIAL E MÉTODOS}

Foram utilizados quatro primatas não humanos, sendo dois machos e duas fêmeas, da espécie Saimiri sciureus, oriundos do Centro Nacional de Primatas de Ananindeua/PA, com diferentes históricos de mortes, não relacionados ao sistema nervoso. Para essa investigação, foram dissecados os dois antímeros de cada animal.

Este estudo está em concordância com o Comitê de Ética em Pesquisa com Animais - CEPAN, do Instituto Evandro Chagas, Ananindeua/PA, sob no. 013/2013.

Os animais foram previamente fixados, com perfusão de solução aquosa de formol a $10 \%$ por meio da artéria carótida comum
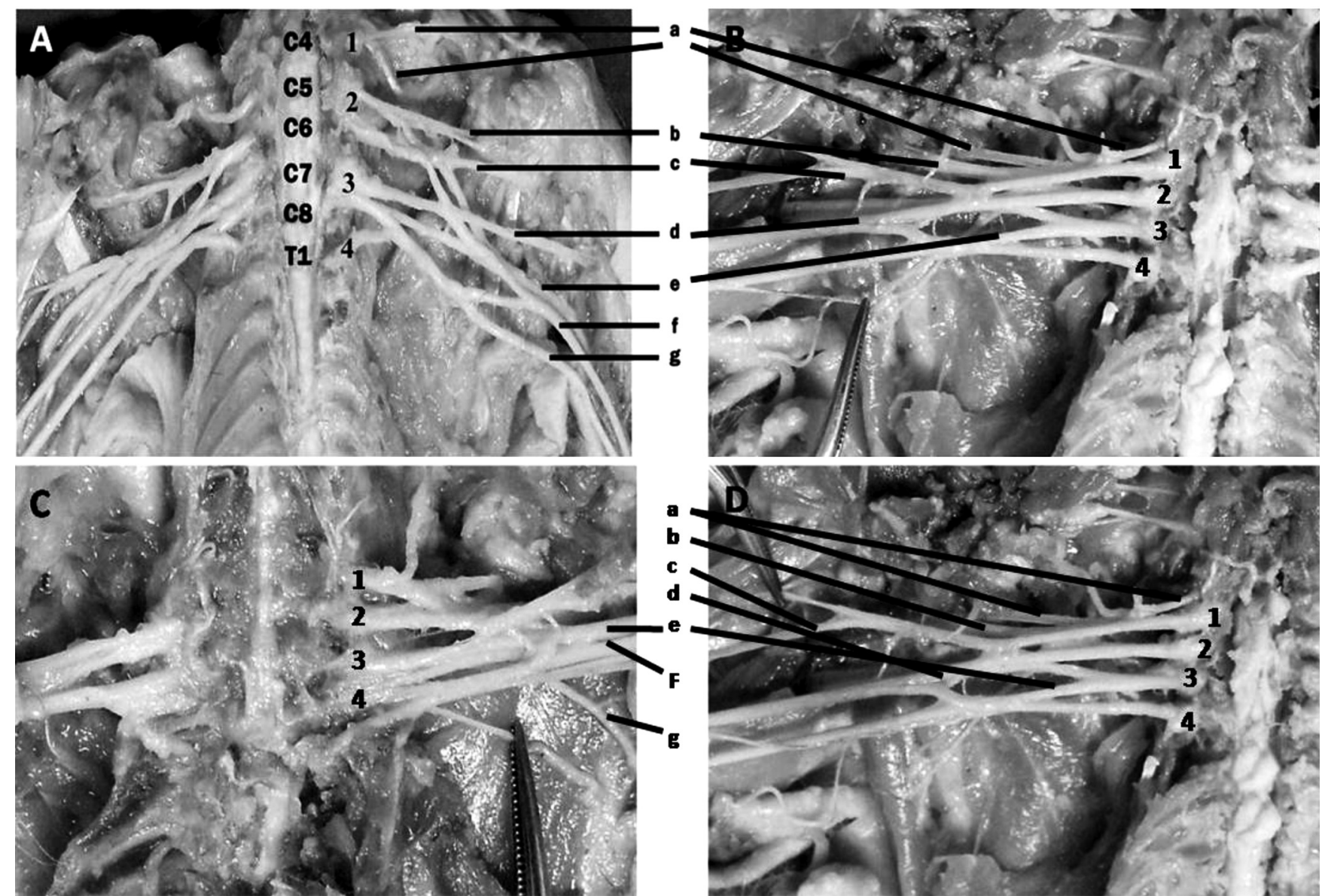

Fig.1.Plexo braquial de Saimiri sciureus, vista ventral contemplando os dois antímeros. (A) Origem dos nervos a partir de C4 a T1. Divisão em 4 troncos: cranial (1), médio-cranial (2), médio-caudal (3), caudal (4). Identificação dos nervos: (a) n. subclávio, (b) n. supraescapular, (c) n. subescapular, (d) n. radial, (e) n. axilar, (f) n. mediano, (g) n. ulnar. (B) (a) n. subclávio, (b) n. supraescapular, (c) n. subescapular, (d) n. radial, (e) n. axilar e n. cutâneo medial do antebraço (pinça). (C) e) n. axilar, (f) n. mediano, (g) n. ulnar e n. toracodorsal (pinça). (D) (a) n. subclávio, (b) n. supraescapular, (c) n. subescapular, (d) n. radial, (e) n. axilar e n. musculocutâneo (pinça). 
direita, abordada por incisão cervical mediana ventral, além de infusões intramusculares e nas cavidades corporais. Após os procedimentos descritos, os espécimes foram mergulhados em recipientes contendo a mesma solução fixadora por período de uma semana e posteriormente dissecados.

Com o uso de materiais básicos de dissecação, foram removidas as vísceras torácicas, assim como, o tecido adiposo da região, expondo parcialmente os nervos oriundos do plexo em estudo. Tal dissecação foi realizada sob técnica de mesoscopia retirando-se primeiramente o esterno e vísceras torácicas, e, em seguida, os corpos vertebrais até a exposição completa da medula espinhal e das raízes formadoras do plexo, sendo possível analisar a origem e a composição dos nervos oriundos do plexo braquial de $S$. sciureus.Toda nomenclatura adotada foi baseada na Nomenclatura Atômica Veterinária (International Committee on Veterinary Gross Anatomical Nomenclature, 2005).

\section{RESULTADOS}

O plexo braquial de Saimiri sciureus, baseado nos quatro exemplares estudados, é constituído por fibras neurais provenientes da união das raízes das vértebras cervicais C4, C5, C6, C7, C8 e T1, e organizado em quatro troncos, o primeiro (cranial) formado pelos nervos espinhais de $\mathrm{C} 4$, o segundo (médio-cranial) por C5 e C6, o terceiro (médio-caudal) por C7 e C8, e o quarto (caudal) originado em T1 (Fig.1A).

Cada um destes troncos originou um nervo ou um grupo de nervos, cuja origem variou de um animal para outro, havendo nervos duplos e nervos com origem dupla, como o observado no nervo subescapular, considerado duplo por ser originário de C5 e C6. Já o nervo radial, variou sua origem em C6 e C7. Os nervos axilar, musculocutâneo e mediano, tiveram a mesma variação de $\mathrm{C} 5$ a C8, enquanto que os nervos ulnar e cutâneo medial do antebraço, variaram de C8 a T1.

$\mathrm{Na}$ maioria dos animais, o primeiro tronco (cranial) originou o nervo subclávio que se dividiu em dois ramos; o segundo tronco (médio-cranial) deu origem aos nervos supraescapular, subescapular, parte do radial (Fig.1A), sendo que em dois animais, o segundo tronco originou os nervos axilar (Fig.1A), musculocutâneo (Fig.1D) e ao mediano (Fig.1A).

O terceiro tronco (médio-caudal) formou parte do nervo radial (Fig.1A), e em dois animais, formou os nervos axilar, musculocutâneo, mediano, toracodorsal (Fig.1C), ulnar (Fig.1A) e cutâneo medial do antebraço (Fig.1B), sendo os dois últimos também originados no quarto tronco (caudal) (Quadro 1).

Quanto aos grupos musculares supridos pelos nervos subclávio, supraescapular, subescapular, radial, axilar, mediano, ulnar, cutâneo medial do antebraço, toracodor-

\section{Quadro 1. Origem e nervos que compõem o plexo braquial de Saimiri sciureus}

\begin{tabular}{cc}
\hline \multicolumn{1}{c}{ Origem } & Nervos \\
\hline C4 (Tronco cranial) & Subclávio \\
C5-C6 (Tronco médio- & Supraescapular, subescapular, parte do radial, axilar, \\
cranial) & musculocutâneo e mediano \\
C7-C8 (Tronco médio- & Parte do radial, axilar, musculocutâneo, mediano, to- \\
caudal) & racodorsal, ulnar e cutâneo medial do antebraço \\
T1 (Tronco caudal) & Ulnar e cutâneo medial do antebraço
\end{tabular}

sal e musculocutâneo, podemos citar respectivamente os seguintes músculos: $\mathrm{m}$. subclávio, mm. supra e infraespinhais, m. subescapular, m. tríceps braquial, m. tensor da fáscia do antebraço, $m$. ancôneo, $m$. redondo maior e menor, $\mathrm{m}$. deltóide, mm. flexores do carpo, pele da face medial do antebraço, m. grande dorsal, m. coracobraquial e m. bíceps braquial (Quadro 2).

Quadro 2. Grupos musculares inervados pelo plexo braquial de Saimiri sciureus

\begin{tabular}{|c|c|}
\hline Nervos & Músculos \\
\hline N. subclávio & m. subclávio \\
\hline N. supraescapular & mm. supra e infraespinhais \\
\hline N. subescapular & m. subescapular \\
\hline N. radial & $\begin{array}{l}\text { m. tríceps braquial, m. tensor da fáscia do antebra- } \\
\text { ço, m. ancôneo }\end{array}$ \\
\hline N. axilar & m. redondo maior e menor, $\mathrm{m}$. deltóide \\
\hline N. mediano & mm. flexores do carpo \\
\hline N. ulnar & mm. flexores do carpo \\
\hline $\begin{array}{l}\text { N. cutâneo medial do an- } \\
\text { tebraço }\end{array}$ & pele da face medial do antebraço \\
\hline N. toracodorsal & m. grande dorsal \\
\hline N. musculocutâneo & m. coracobraquial e m. bíceps braquial \\
\hline
\end{tabular}

\section{DISCUSSÃO}

O plexo braquial de Saimiri sciureus é bastante semelhante ao de outras espécies de primatas já estudados, porém, apesar de Mizuno (1969) afirmar que nesta mesma espécie o plexo braquial tem início em C5, pudemos constatar que na verdade, tal estrutura tem origem à partir de $\mathrm{C} 4$, assim como observado em chimpanzés e gorilas (Koizumi \& Sakai 1995), podendo ocorrer de forma acentuada em Macaca (Macacus cyclopsis) (Brooks 1883), macaco prego (Cebus apella) (Ribeiro et al. 2005) e orangotango (Pongo sp.) (Kawashima et al. 2007), o que difere dos registros sobre o plexo braquial de gálalo (Galago senegalensis) (Kanagasuntheram \& Mahran 1960), babuíno (Papio ursinus) (Booth et al. 1997) e macaco-barrigudo (Lagothrix sp.) (Cruz \& Adami 2010), que inicia-se em C5.

Essa variação se dá durante o processo evolutivo, no qual o plexo braquial descola-se cranialmente, atingindo C4 nos símios e C3 em humanos (Parada et al. 1989). Tal estrutura recebe contribuição principal dos três últimos nervos espinhais cervicais e do primeiro torácico nos animais domésticos (Dyce 2004). A participação de ramos mais craniais, como C4 e C5, caracteriza um plexo pré-fixado, como o observado em macacos do novo mundo (Sugiyama 1965, Hill 1972), como é o caso de Saimiri sciureus.

Em gálago (Galago senegalensis), guenon (Cercopithecus), babuíno (Papio ursinus), Macaco Gibão (Hylobates sp.), gorila, orangotango, chimpanzé, macaco barrigudo (Lagothrix sp.), macaco prego (Cebus apella), e até mesmo no humano, o plexo braquial é constituído por apenas três troncos: cranial (superior), formado por C5 e C6, médio, formado por C7 e caudal (inferior), formado por C8 e T1 (ocasionalmente com participação de T2) (Kanagasuntheram \& Mahran 1960, Sugiyama 1965, Hill 1966, Koizumi \& Sakai 1995, Booth et al. 1997, Oliveira et al. 2003, Narahashi et al. 2005, Ribeiro et al. 2005, Cruz e Adami 2010). Tal situação é bastante diferente no macaco-de-cheiro (Sai- 
miri sciureus), pois devido à participação direta de C4, há formação de quatro troncos, com localizações bem precisas, sendo o primeiro (cranial) em C4, o segundo (médio-cranial) em C5 e C6, o terceiro (médio-caudal) em C7 e C8 e o quarto (caudal) em T1.

De forma geral, em S. sciureus, o tronco cranial deu origem ao nervo subclávio; fato relatado apenas por Ribeiro (2002) em uma pequena amostra de grupo de animais de avaliados (Cebus apella).

0 tronco médio-cranial deu origem aos nervos supraescapular, subescapular, parte do radial, e em alguns casos, aos nervos axilar, musculocutâneo e ao mediano. Estes mesmos nervos, apesar da diferença na quantidade de troncos, comportaram-se de forma semelhante, no chimpanzé (Troglotydes niger), anubis (Cynocephalus anubis), babuínos (Papio ursinus) e em Lagothrix sp. os quais originaram-se basicamente no tronco cranial e médio e por vez o musculocutâneo originou-se no tronco caudal (Champneys 1975, Booth et al. 1997, Cruz \& Adami 2010).

No que tange o tronco médio-caudal, este ocasionou nervo radial, e em alguns animais, os nervos axilar, musculocutâneo, mediano, toracodorsal, ulnar e cutâneo medial do antebraço, porém, os dois últimos também foram originados do tronco caudal. Tal avaliação assemelha-se aos achados de Cruz \& Adami (2010), todavia, há uma pequena dissonância quanto nervo musculocutâneo, que no macaco barrigudo tem origem em tronco cranial.

\section{CONCLUSÕES}

Diferentemente do que ocorre nos primatas já estudados, o plexo braquial de Saimiri sciureus é oriundo dos nervos espinhais ao nível de $\mathrm{C} 4$ a T1 constituindo quarto troncos sendo eles: tronco cranial, tronco médio-cranial, tronco médio-caudal e tronco caudal.

Os nervos originados destes quatro troncos possuem origem e trajetória semelhantes de outros primatas, havendo pequenas variações.

Os resultados desta investigação científica são salutares, para melhor compreensão dos processos clínicos e cirúrgicos, que envolvam principalmente questões de motricidade na espécie avaliada.

Agradecimentos.- Ao Centro Nacional de Primatas (Cenp) de Ananindeua/Pará, Brasil, por fornecer os animais, e à Universidade Federal Rural da Amazônia, através do PIBIC-REUNI/UFRA, por financiar o desenvolvimento deste estudo.

\section{REFERÊNCIAS}

Auricchio P. 1995. Primatas do Brasil. Terra Brasilis, São Paulo. 168p. Aversi-Ferreira T., Silva M.S.L., Paula J.P., Silva L.G. \& Silva N.P. 2005. Ana- tomia comparativa dos nervos do braço de Cebus apella: descrição do músculo dorsoepitroclear. Acta Sci. Biol. Sci. 27(3):291-296.

Barcellos J.F. M., Branco E., Fioretto E.T., Imbeloni A.A., Muniz J.A.P.C. \& Lima A.R. 2011. Macroscopic aspects of Saimiri sciureus dura mater. Pesq. Vet. Bras. 31(Supl.1):7-10.

Booth K.K., Baloyi F.M. \& Lukhele O.M. 1997. The brachial plexus in the Chacma baboon (Papio ursinus). J. Med. Primatol. 26:196-203.

Brooks W.T. 1883. The brachial plexus of the Macaque monkey and its analogy with that of man. J. Anat. Physiol. 17(3):329-332.

Champneys F. 1975. On the muscles and nerve of a Chimpanzee (Troglodytes niger) and Cynocephalus anubis. J. Anat. Phys. 6(1):176-211.

Cruz G.A.M., Adami M. 2010. Anatomia do plexo braquial de macaco-barrigudo (Lagothrix lagothricha). Pesq. Vet. Bras. 30(10):881-886.

Dukelow W.R. 1978. Reproduction in the squirrel monkey (Saimiri sciureus). Rec. Adv. Primatol. 2:195-200.

Evans H.E. \& Lahunta A. 1994. Abdome, pelve e membro pélvico, p.118119. In: Ibid. (Eds), Guia para a Dissecação do Cão. 3ª ed. Edwards Brothers, Ithaca.

Frandson R.D., Wylke W.L. \& Fails A.D. 2005. Anatomia e Fisiologia dos Animais de Fazenda. 6 ${ }^{\mathrm{a}}$ ed. Guanabara Koogan, Rio de Janeiro.

Getty R. 1986. Sisson and Grossman's Anatomy of the Domestic Animals. $5^{\text {th }}$ ed. W.B. Saunders Company, Philadelphia. 1211p.

Hill W.C.O. 1966. Primates: Comparative anatomy and taxonomy. VI. Catarrhini: Cercopithecoidea-Cercopithecinae. Edinburgh University Press, Edinburgh. 757p.

Hill W.C.O. 1972. Primates: Comparative anatomy and taxonomy. V. Cebidae: Part B. Edinburgh University Press, Edinburgh. 537p.

International Committee on Veterinary Gross Anatomical Nomenclature 2005. Nomina Anatomica Veterinaria. $5^{\text {th }}$ ed. Hannover. 166p.

Kanagasuntheram R. \& Mahran Z.Y. 1960. Observations on the nervous system of the lesser bush baby (Galago senegalensis senegalensis). J. Anat. 94(4):512-527.

Koizumi M. \& Sakai T. 1995. The nerve supply to coracobrachialis in apes. J. Anat. 186(2):395-403.

Mizuno N. 1969. The brachial plexus in the Squirrel Monkey (Saimiri sciureus). Primates 10:19-35.

Narahashi E., Caldana W.C.I., Zoner C., Honda E., Caporrino F.A., Mine F., Yamada V.L., Amaral D.T., Natour J. \& Fernandes A.R.C. 2005. Diagnóstico por imagem do plexo braquial. Revta Bras. Reumatol. 45(4):245-249.

Oliveira M.F., Miglino M.A., Passipieri M., Teixeira D.G., Moura C.E.B., Martins D.S., Benedicto H., Thomaz J.M. \& Ambrósio C.E. 2003. Anatomia do plexo braquial do macaco Gibão (Hylobates sp.). Int. J. Morphol. 21(1):59.

Ribeiro A.R. 2002. Estudo anatômico do plexo braquial do macaco Cebus apella: origem, composição e nervos resultantes. Dissertação de Mestrado em Anatomia dos Animais Domésticos, Faculdade de Medicina Veterinária e Zootecnia, USP, São Paulo. 146p.

Ribeiro A.R., Prada I.L.S., Silva Z., Barros R.A.C. \& Silva D.C.O. 2005. Origem do plexo braquial do macaco Cebus apella. Braz.J. Vet. Res. Anim. Sci. 42(2):143-149.

Sugiyama T. 1965. On the plexus brachialis of Macacus cyclopsis. Acta Med. Nagasaki 9(3):65-68.

Vaughan T.A. 1985. Order primates, p.138-143, In: Ibid. (Ed.), Journal of Mammalogy. Northern Arizona University, Flagstaff. 